\title{
Granular computing, rough entropy and object extraction
}

\author{
Sankar K. Pal ${ }^{\text {a,1 }}$, B. Uma Shankar ${ }^{\mathrm{a}, *}$, Pabitra Mitra ${ }^{\mathrm{b}}$ \\ ${ }^{a}$ Machine Intelligence Unit, Indian Statistical Institute, Calcutta 700 108, India \\ ${ }^{\mathrm{b}}$ Department of Computer Science and Engineering, Indian Institute of Technology, Kanpur 208 016, India \\ Received 4 August 2004 \\ Available online 28 July 2005 \\ Communicated by W. Pedrycz
}

\begin{abstract}
The problem of image object extraction in the framework of rough sets and granular computing is addressed. A measure called "rough entropy of image" is defined based on the concept of image granules. Its maximization results in minimization of roughness in both object and background regions; thereby determining the threshold of partitioning. Methods of selecting the appropriate granule size and efficient computation of rough entropy are described.
\end{abstract}

(C) 2005 Elsevier B.V. All rights reserved.

Keywords: Rough sets; Entropy; Image segmentation; Set approximation; Granules

\section{Introduction}

It has been argued, both from philosophical and theoretical points of views, that information granulation is essential to human problem solving, and hence has very significant impact on the design and implementation of intelligent system. Zadeh

\footnotetext{
* Corresponding author. Tel.: +91 33 25753107; fax: +91 33 25783357.

E-mail addresses: sankar@isical.ac.in (S.K. Pal), uma@) isical.ac.in (B. Uma Shankar), pmitra@iitk.ac.in (P. Mitra).

${ }^{1}$ Currently with the US Naval Research Laboratory, Washington, DC, USA.
}

(1997) identified three basic concepts that underlie the process of human cognition, namely, granulation, organization, and causation. "Granulation involves decomposition of whole into parts, organization involves integration of parts into whole, and causation involves association of causes and effects".

A granule is a clump of objects (points), in the universe of discourse, drawn together by indistinguishability, similarity, proximity, or functionality. Granulation leads to information compression/summarization. In situations involving incomplete, uncertain, or vague information, it may be difficult to differentiate different elements 
and instead it is convenient to consider granules, i.e., clump or group of indiscernible elements, for performing operations. Granular computing ( GrC) may be regarded as a unified framework for theories, methodologies and techniques that make use of such granules in the process of problem solving.

Recently, rough set theory (Pawlak, 1991) has become a popular mathematical framework for granular computing. The focus of rough set theory is on the ambiguity caused by limited discernibility of objects in the domain of discourse. Its key concepts are those of object 'indiscernibility' and 'set approximation'. The primary use of rough set theory has so far mainly been in generating logical rules for classification and prediction (Skowron and Rauszer, 1992) using information granules; thereby making it a prospective tool for pattern recognition, image processing, feature selection, data mining and knowledge discovery process from large data sets. Use of rough set rules based on reducts has significant role for dimensionality reduction/feature selection by discarding redundant features; thereby having potential application for mining large data sets (Komorouski et al., 1999). As far as rough set theoretic image processing is concerned, there is hardly any investigation reported so far. However, in related areas like pattern analysis/clustering mention may be made of the studies of Wojcik (1987), and Pal and Mitra (2002). While rough sets are used in (Wojcik, 1987) for describing image features for analysis, they are used in a part in (Pal and Mitra, 2002) for initializing EM algorithm in conjunction with minimal spanning tree (MST) for clustering with application to multi spectral images.

In the present article we demonstrate an application of rough sets and granular computing for object extraction (Gonzalez and Woods, 2002) from gray scale image. In gray scale images boundaries between object regions are often ill-defined. This uncertainty can be handled by describing the different objects as rough sets with upper (outer) and lower (inner) approximations. The set approximation capability of rough sets is exploited in the present investigation to formulate an entropy measure, called rough entropy, quantifying the uncertainty in an object-background image. This has been done by defining an image as a collection of pixels and the equivalence relation induced partition as pixels lying within each non-overlapping window over the image. With this definition the roughness of various transforms (or partitions) of the image can be computed using image granules, i.e., windows, of different sizes.

Maximization of the said rough entropy measure minimizes the uncertainty arising from vagueness of the boundary region of the object. Therefore, for a given granule size, the threshold for object-background classification can be obtained through its maximization with respect to different image partitions. A guideline for selecting the appropriate granule size from gray level distribution is given, as well as a way of computing the rough entropy efficiently only in one pass (or scan) of the image. Effectiveness of the method is demonstrated on different kinds of images.

\section{Rough entropy measure of an image}

\subsection{Rough set}

Let $\mathscr{A}=\langle U, A\rangle$ be an information system, and let $B \subseteq A$ and $X \subseteq U$. We can approximate the set $X$ using only the information contained in $B$ by constructing the lower and upper approximations of $X$. If $X \subseteq U$, the sets $\left\{\boldsymbol{x} \in U:[\boldsymbol{x}]_{B} \subseteq X\right\}$ and $\left\{\boldsymbol{x} \in U:[\boldsymbol{x}]_{B} \cap X \neq \emptyset\right\}$, where $[\boldsymbol{x}]_{B}$ denotes the equivalence class of the object $x \in U$ relative to $I_{B}$ (the equivalence relation), are called the $B$ lower and B-upper approximations of $X$ in $U$. They are denoted by $\underline{B} X$ and $\bar{B} X$, respectively. The objects in $\underline{B} X$ can be certainly classified as members of $X$ on the basis of knowledge in $B$, while objects in $\bar{B} X$ can only be classified as possible members of $X$ on the basis of $B$. These are illustrated in Fig. 1 where the sets of dark-gray granules represent lower approximation, while those of both darkgray and light-gray granules together denote upper approximation. Therefore, a rough set is nothing but a crisp set with rough representation.

The roughness of a set $X$ with respect to $B$ can be characterized numerically (Pawlak, 1991) as $R_{\alpha}=1-\frac{|B X|}{|\bar{B} X|}$. This means if roughness of the set $X$ is 0 then $X$ is crisp with respect to $B$, and if 


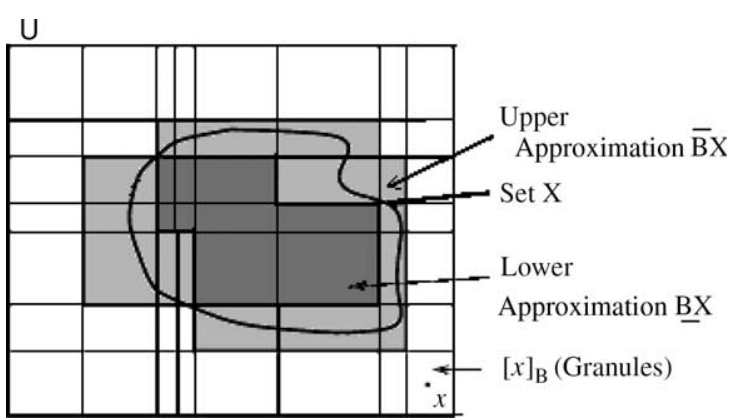

Fig. 1. Rough representation of a set with upper and lower approximations.

$R_{\alpha}>0$ then $X$ is rough (i.e., $X$ is vague with respect to $B$ ). For details one may refer to Pawlak (1991), Skowron and Rauszer (1992) and Komorouski et al. (1999).

\subsection{Image as a rough set}

In gray scale images boundaries between object regions are often ill defined because of grayness and/or spatial ambiguities (Pal, 2001). This uncertainty can be handled by describing the different objects as rough sets with upper (or outer) and lower (or inner) approximations. Here the concepts of upper and lower approximation can be viewed, respectively, as outer and inner approximations of an image region in terms of granules.

Let the universe $U$ be an image consisting of a collection of pixels. Then if we partition $U$ into a collection of non-overlapping windows (of size $m \times n$, say), each window can be considered as a granule $G$. In other words, the induced equivalence classes $I_{m \times n}$ have $m \times n$ pixels in each non-overlapping window. Given this granulation, object regions in the image can be approximated by rough sets.

Let us consider an object-background separation (a two class) problem of an $M \times N, L$ level image. Let $\operatorname{prop}(B)$ and $\operatorname{prop}(O)$ represent two properties (say, gray level intervals $0,1, \ldots, T$ and $T+1, T+2, \ldots, L-1)$ that characterize background and object regions, respectively. Given this framework, object and background can be viewed as two sets with their rough representation as follows:
The inner approximation of the object $\left(\underline{O}_{T}\right)$ :

$$
\begin{aligned}
\underline{O}_{T}= & \left\{\bigcup_{i} G_{i} \mid P_{j}>T, \forall j=1, \ldots, m n, \quad\right. \text { and } \\
& \left.P_{j} \text { is a pixel belonging to } G_{i}\right\} .
\end{aligned}
$$

Outer approximation of the object $\left(\bar{O}_{T}\right)$ :

$$
\begin{aligned}
\bar{O}_{T}= & \left\{\bigcup_{i} G_{i}, \exists j, j=1, \ldots, m n \text { s.t. } P_{j}>T,\right. \\
& \text { where } \left.P_{j} \text { is a pixel in } G_{i}\right\} .
\end{aligned}
$$

Inner approximation of the background $\left(\underline{B}_{T}\right)$ :

$$
\begin{aligned}
\underline{B}_{T}= & \left\{\bigcup_{i} G_{i} \mid P_{j} \leqslant T, \forall j=1, \ldots, m n, \quad\right. \text { and } \\
& \left.P_{j} \text { is a pixel belonging to } G_{i}\right\} .
\end{aligned}
$$

Outer approximation of the background $\left(\bar{B}_{T}\right)$ :

$$
\begin{aligned}
\bar{B}_{T}= & \left\{\bigcup_{i} G_{i}, \exists j, j=1, \ldots, m n \text { s.t. } P_{j} \leqslant T,\right. \\
& \text { where } \left.P_{j} \text { is a pixel in } G_{i}\right\} .
\end{aligned}
$$

Therefore, the rough set representation of the image (i.e., object $O_{T}$ and background $B_{T}$ ) for a given $I_{m \times n}$ depends on the value of $T$.

Let the roughness of object $O_{T}$ and background $B_{T}$ be defined as

$$
\begin{aligned}
& R_{O_{T}}=1-\frac{\left|\underline{O}_{T}\right|}{\left|\bar{O}_{T}\right|}=\frac{\left|\bar{O}_{T}\right|-\left|\underline{O}_{T}\right|}{\left|\bar{O}_{T}\right|}, \\
& R_{B_{T}}=1-\frac{\left|\underline{B}_{T}\right|}{\left|\bar{B}_{T}\right|}=\frac{\left|\bar{B}_{T}\right|-\left|\underline{B}_{T}\right|}{\left|\bar{B}_{T}\right|},
\end{aligned}
$$

where $\left|\underline{O}_{T}\right|$ and $\left|\bar{O}_{T}\right|$ are the cardinality of the sets $\underline{O}_{T}$ and $\bar{O}_{T}$, and $\left|\underline{B}_{T}\right|$ and $\left|\bar{B}_{T}\right|$ are the cardinality of the sets $\underline{B}_{T}$ and $\bar{B}_{T}$, respectively.

\subsection{Rough entropy measure}

Rough entropy (RE) of an image can be defined as

$\mathrm{RE}_{T}=-\frac{e}{2}\left[R_{O_{T}} \log _{e}\left(R_{O_{T}}\right)+R_{B_{T}} \log _{e}\left(R_{B_{T}}\right)\right]$. 


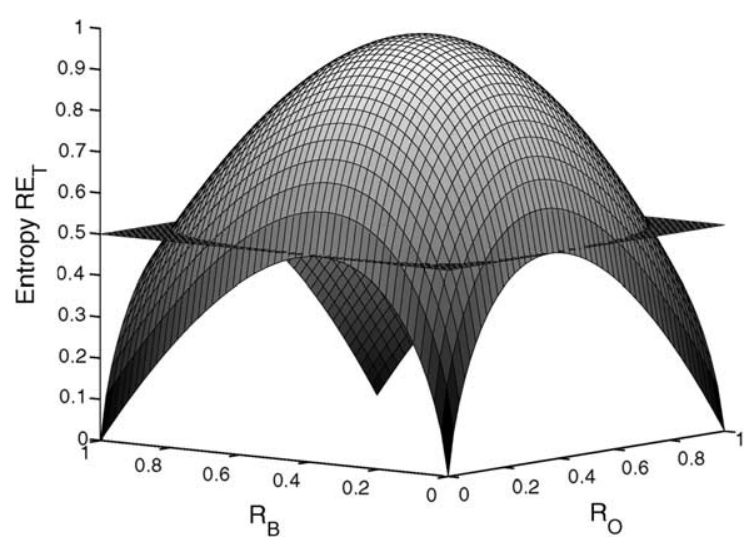

Fig. 2. Plot of rough entropy for various values of roughness of the object and background.

Its plot for various values of $R_{O_{T}}$ and $R_{B_{T}}$ is shown in Fig. 2.

(1) The value of $\mathrm{RE}_{T}$ lies between 0 and 1 .

(2) $\mathrm{RE}_{T}$ has a maximum value of unity when $R_{O_{T}}=R_{B_{T}}=1 / e$, and minimum value of zero when $R_{O_{T}}, R_{B_{T}} \in\{0,1\}$.

(3a) Since the boundary pixels are common for both object and background, we have $\bar{O}_{T}-\underline{O}_{T}=\bar{B}_{T}-\underline{B}_{T}=Q_{T}$, say. Therefore,

$R_{O_{T}}=R_{B_{T}}, \quad$ iff $\left|\underline{O}_{T}\right|=\left|\underline{B}_{T}\right|$.

Under this condition, the distribution of $\mathrm{RE}_{T}$ on the diagonal (joining $(0,0)$ and $(1,1))$ is shown in Fig. 3, where $\mathrm{RE}_{T}$ attains a maximum value of unity at $R_{O_{T}}=$ $R_{B_{T}}=1 / e$.

(3b) When $\left|\underline{O}_{T}\right|<\left|\underline{B}_{T}\right|$, then $R_{O_{T}}>R_{B_{T}} \quad$ and $\left|\underline{O}_{T}\right|>\left|\underline{B}_{T}\right|$, then $R_{O_{T}}<R_{B_{T}}$.

In either case, $\mathrm{RE}_{T}$ will decrease from its maximum value of unity and will reach a value of zero at $(0,0),(0,1),(1,0)$ and $(1,1)$ in the $\left(R_{O_{T}}, R_{B_{T}}\right)$ plane (Fig. 2).

\section{Object extraction minimizing roughness}

Let us describe a method of object enhancement/extraction based on the principle of minimizing the roughness of both object and background regions, i.e., maximizing $\mathrm{RE}_{T}$. As explained in Section 2.2, one can compute for every $T$ the $\mathrm{RE}_{T}$ of

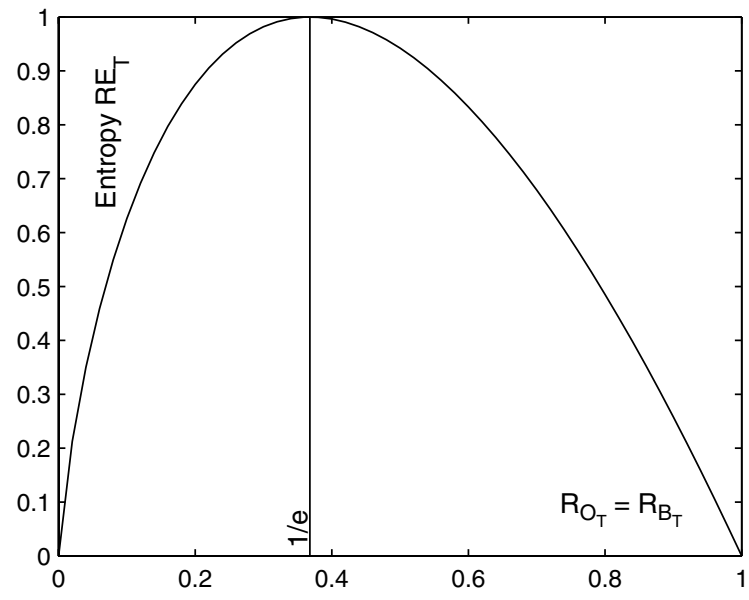

Fig. 3. Plot of rough entropy for the values $(0,0)$ to $(1,1)$ on the diagonal of Fig. 2 (i.e., when $R_{O_{T}}=R_{B_{T}}$ ).

the image, representing the background and object regions $(0, \ldots, T)$ and $(T+1, \ldots, L-1)$, respectively, and select the one for which $\mathrm{RE}_{T}$ is maximum. In other words, select

$T^{*}=\arg \max _{T} \mathrm{RE}_{T}$,

as the optimum threshold to provide the objectbackground segmentation. Note that maximizing the rough entropy to get the required threshold basically implies minimizing both the object roughness and background roughness.

\subsection{Choice of granule size}

As can be seen, the determination of $T^{*}$ by maximization of rough entropy or minimization of roughness depends on the granule size. A choice of granule size can be made from gray level distribution of the image by selecting a value approximately equal to the minimum of half the width of base regions corresponding to all the peaks in the histogram. This will allow the algorithm to take into account the local information (details) of all the regions, as indicated by different peaks in the histogram, and facilitate the detection of the smallest region. Any granule larger (or smaller) than this may result in losing some desirable regions (or detection of spurious undesirable regions) by the decrease (or increase) in the value 
of $T^{*}$, assuming that the regions of interest correspond to lower side of the histogram. The details of the selection procedure are shown in Section 4 with histograms of different images.

\subsection{Algorithm for threshold selection}

Following is the algorithm for efficient implementation of the aforesaid methodology for selecting $T^{*}$.

Let max_gray and min_gray be the maximum and minimum gray level values of the image, respectively. Let granule $i$ represent a window of $m \times n$ pixels. Let total number of granules be total_no_granule.

Initialize: Four integer arrays namely object_ lower, object_upper, background_lower, background_upper each of size (max _gray - min _gray+ 1) to zero.

Step 1: for $i=1$ to total_no_granule max_granule $_{i}=$ maximum gray value of pixels in granule $_{i}$

min_granule $_{i}=$ minimum gray value of pixels in granule $_{i}$

(a) for max_granule $\leqslant \leqslant j \leqslant$ max_gray object_lower $(j)=$ object_lower $(j)+1$

(b) for min_granule $_{i} \leqslant j \leqslant$ max_gray object_upper $(j)=$ object_upper $(j)+1$

(c) for min_gray $\leqslant j \leqslant$ min_granule ${ }_{i}$ background_lower $(j)=$ background lower $(j)+1$

(d) for min_gray $\leqslant j \leqslant$ max_granule $_{i}$ background_upper $(j)=$ background_ upper $(j)+1$

Step 2: for $l=$ min_gray to max_gray object_roughness $(l)=1-[\operatorname{object} \operatorname{lower}(l) /$

background_roughness $(l)$ object_upper $(l)]$ $=1-[$ background_lower $(l) /$ background_upper $(l)]$

Rough_entropy $(l)$ $=-\left[\frac{e}{2}\right] \times[$ object_roughness $(l)$ $\log _{e}($ object_roughness $(l))$ + background_roughness $(l)$ $\log _{e}$ (background_roughness $\left.\left.(l)\right)\right]$

Step 3: Threshold(optimal)

$$
=\underset{l}{\arg \max }[\text { rough_entropy }(l)] \text {. }
$$

Remark. Given the max_gray and min_gray values, the computation of rough entropy (and hence the algorithm) requires only a single scan of pixels in the image, since max_granule ${ }_{i}$ and min_granule ${ }_{i}$ are computed exactly once for each $i$. Therefore the computational complexity of the algorithm is same as that of histogram computation.

\section{Experimental results}

The effectiveness of the methodology for object extraction based on rough entropy (Eq. (2)) is demonstrated on three different types of images. These are: (i) a text image (TEXT), (ii) a blocks image (BLOCKS), and (iii) a remote sensing image (CALCUTTA). The details of the images are as follows: The TEXT image (Fig. 7(a)) is of $1003 \times 2249$ pixels and scanned from a news letter of IAPR. The BLOCKS image (Fig. 8(a)) is taken with a CCD camera when the blocks of different shapes are placed on table and imaged. The size of the image is $512 \times 512$ pixels. The CALCUTTA image is an IRS-1A image, which was taken using the scanner LISS-II (Linear Imaging Self Scanner) in the range of wavelength $0.77-0.86 \mu \mathrm{m}$ and it has a spatial resolution of $36.25 \mathrm{~m} \times 36.25 \mathrm{~m}$. The image is covering an area around the city of Calcutta (Fig. 9(a)). The image is of size $512 \times 512$. Due to poor illumination, the actual object classes

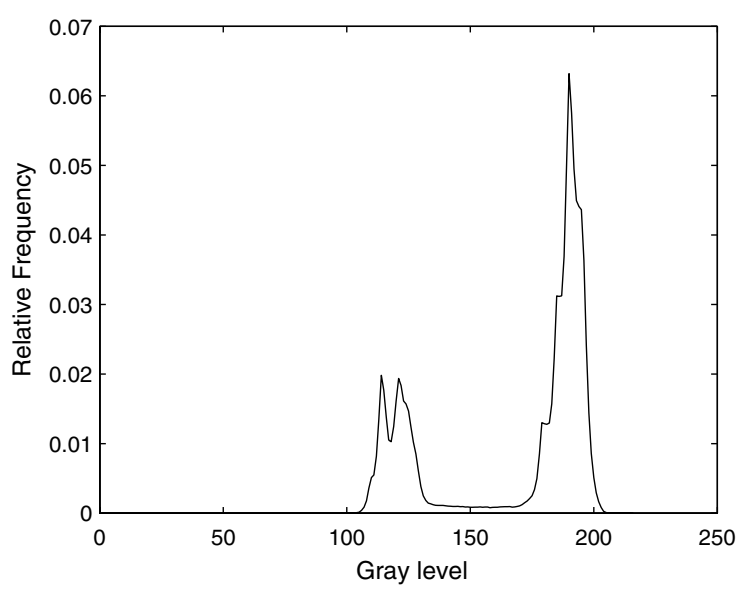

Fig. 4. Histogram of the TEXT image, minimum estimated base width is 30 between gray levels 105 and 135 . 


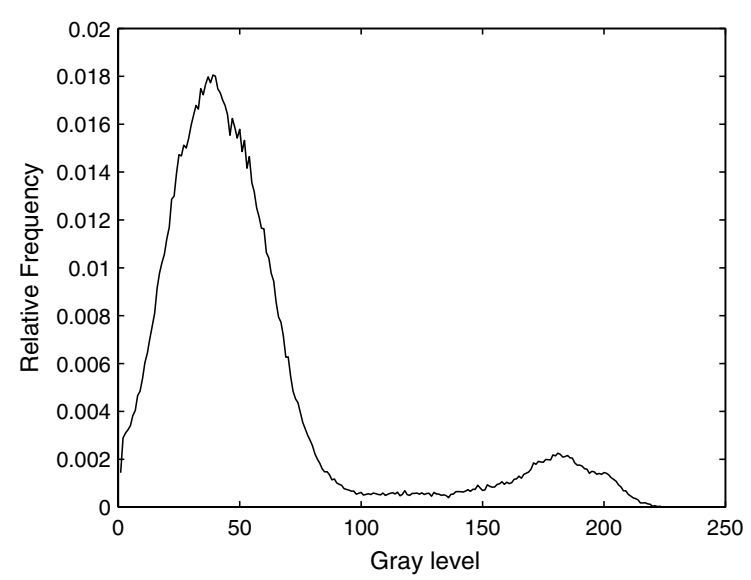

Fig. 5. Histogram of the BLOCKS image, minimum estimated base width is 68 between gray levels 150 and 218 .

present in the input image are not visible clearly; therefore an enhanced version of the input image highlighting the different object regions are shown in Fig. 9(a) for convenience.

At first we discuss about the selection of the granule size (window size) for the computation of rough entropy on these images. The histograms (Figs. 7-9) of all these images are bimodal. For

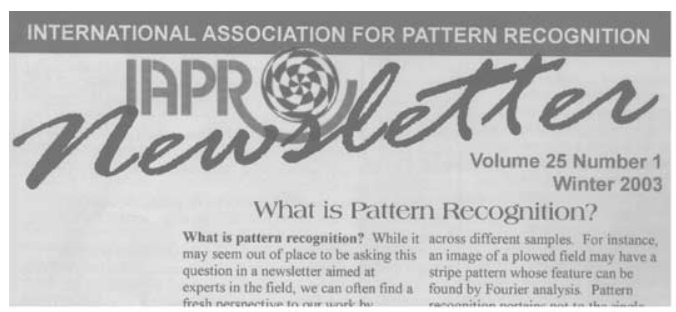

a

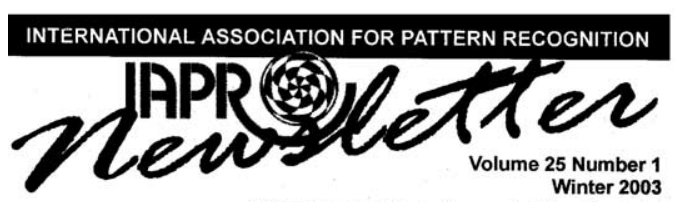

What is Pattern Recognition?

What is pattern recognition? While it across different samples. For instance, may seem out of place to be asking this an image of a plowed field may have $\begin{array}{ll}\text { question in a newsiteter aimed at } & \text { stripe pattern whose feature can be } \\ \text { experts in the field, we can often find a found by Fourier analysis. Patterm }\end{array}$

c

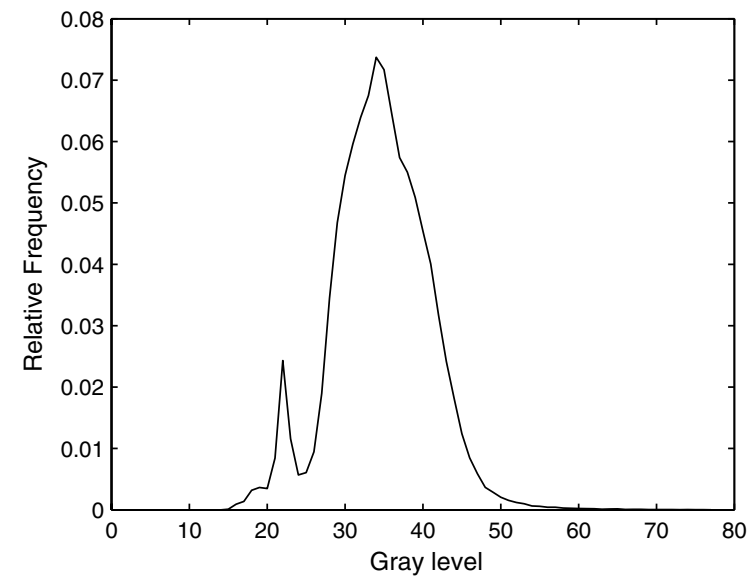

Fig. 6. Histogram of the CALCUTTA image, minimum estimated base width is 8 between 16 and 24 gray level.

the TEXT image (Fig. 4) the base width of each region is approximately 30 levels. Therefore, as mentioned in Section 3, we take the granule size as $15 \times 15$ (half of the smaller base width).

Similarly for the BLOCKS image (Fig. 5) the widths of the regions at the base are 100 and 68 approximately. Therefore, a granule for this image can be of size $34 \times 34$. For the CALCUTTA image

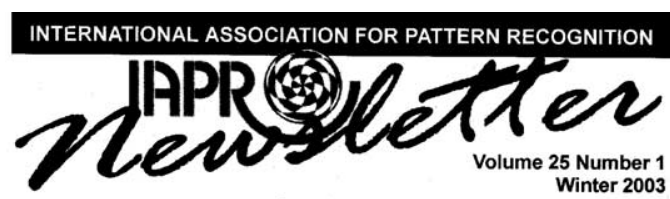

What is Pattern Recognition?

What is pattern recognition? While it across different samples. For instance, may seem out of place to be asking this an image of a plowed field may have a $\begin{array}{ll}\text { question in a newsletter aimed at } & \text { stripe pattern whose feature can be } \\ \text { experts in the field, we can offen find a found by Fourier analysis. Pattern }\end{array}$

b

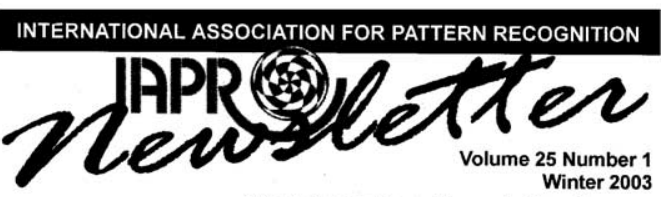

What is Pattern Recognition?

What is pattern recognition? While it across different samples. For instance, may sent out of place to be asking this an image of a plowed field may have a

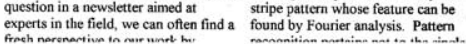

d

Fig. 7. Thresholds by maximizing rough entropy using granule of different sizes on TEXT image: (a) original, (b) threshold $=169$, granule size $=15 \times 15$, (c) threshold $=171$, granule size $=10 \times 10$, (d) threshold $=168$, granule size $=19 \times 19$. 
(Fig. 6) the widths being 8 and $30,4 \times 4$ is a choice of the granule size.

Experiments were conducted with these granular sizes, as well as with other values, to demonstrate the effectiveness of the aforesaid selection criterion. The results are as follows.

The image TEXT is seen to be clearly segmented into text and back ground portions as shown in Fig. 7(b), with threshold value 169 which is determined with granule size of $15 \times 15$. The other two results which are obtained with window sizes $10 \times 10$ and $19 \times 19$ are shown in Fig. $7(\mathrm{c})$ and (d) corresponding to thresholds 171 and 168. As explained in Section 3.1, increase in granule size is seen to reduce the value of $T^{*}$, and vice versa. Since the histogram has a wide smooth flat valley between two major peak regions, the resulting threshold image does not have significant visual change even for a large variation of granule size from $10 \times 10$ to $19 \times 19$.

For the image BLOCKS, the algorithm is able to partition the blocks as object from background, with a threshold at 99 obtained with granule size of $34 \times 34$ (Fig. 8(b)). Increase (or decrease) in granule size, as expected, decreases (or increases) the value of $T^{*}$ (e.g., 86 for Fig. 8(c) and 104 for Fig. $8(\mathrm{~d})$ corresponding to granules of $36 \times 36$ and $29 \times 29$ ). Since the object regions (blocks) here correspond to upper part of the histogram, the decrease in the $T^{*}$ value from 99 to 86 resulted in the detection of more spurious regions (dots). On the other hand, increase in $T^{*}$ value from 99 to 104 almost on the flat valley could not make significant change in the output image.

Unlike the above two images, the image CALCUTTA has many more object regions. However,

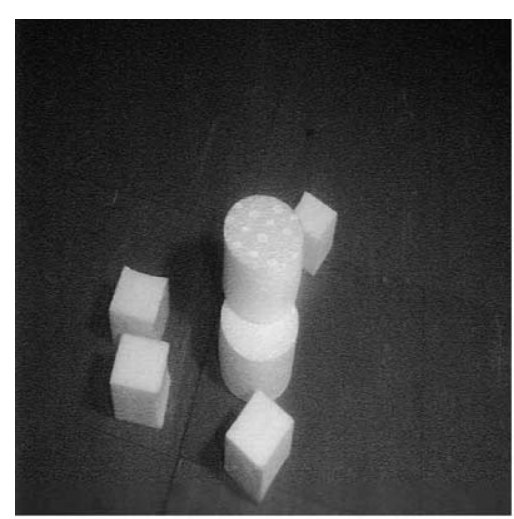

a

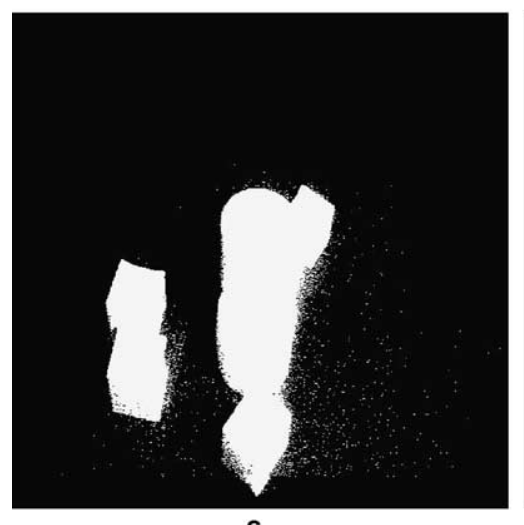

c

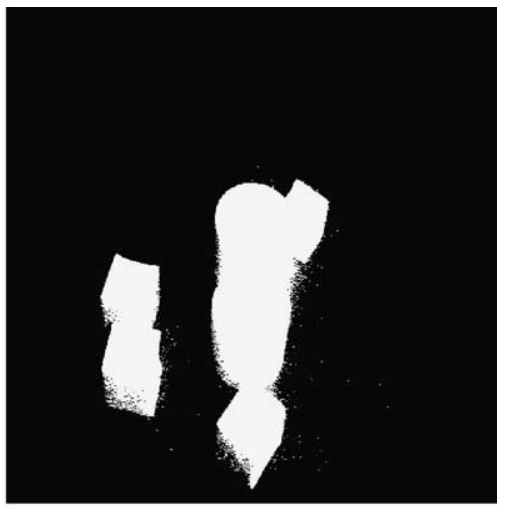

b

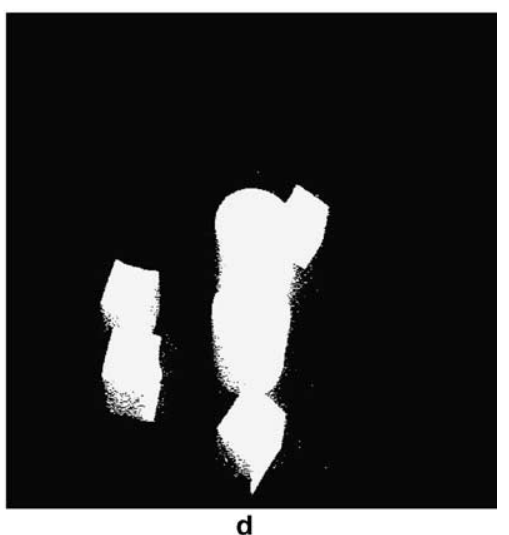

Fig. 8. Thresholds by maximizing rough entropy using granule of different sizes on BLOCKS image: (a) original, (b) threshold $=99$, granule size $=34 \times 34$, (c) threshold $=86$, granule size $=36 \times 36$, (d) threshold $=104$, granule size $=29 \times 29$. 


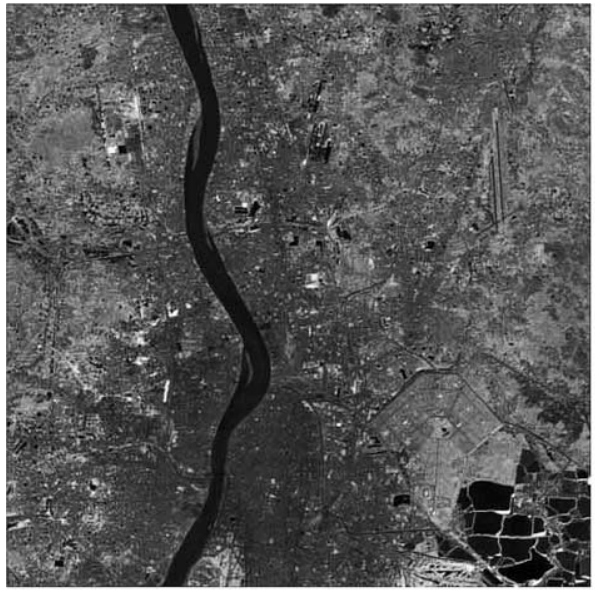

a

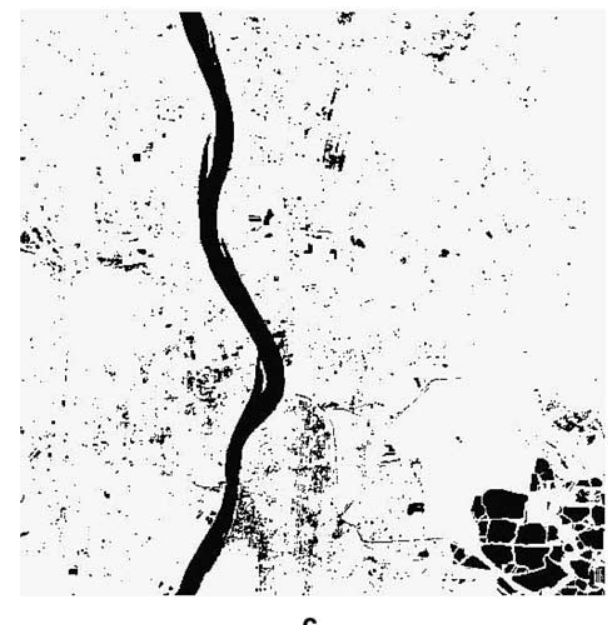

c

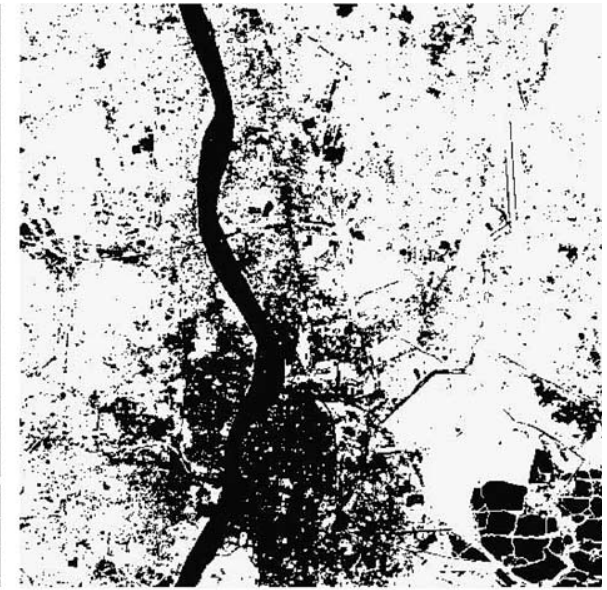

b

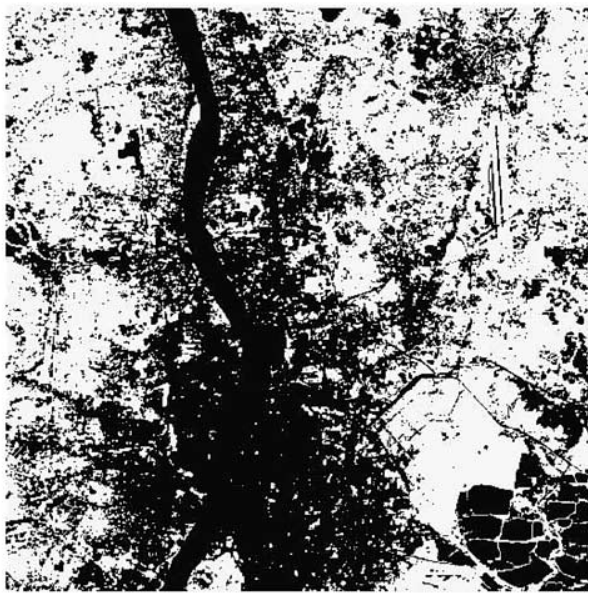

d

Fig. 9. Thresholds by maximizing rough entropy using granule of different sizes on CALCUTTA image: (a) original, (b) threshold $=30$, granule size $=4 \times 4$, (c) threshold $=26$, granule size $=6 \times 6$, (d) threshold $=33$, granule size $=2 \times 2$.

considering it as a two class (object and background) problem, different segmented results are shown. The output shown in Fig. 9(b) corresponds to granule size of $4 \times 4$ which produced a threshold of 30. The other two outputs, Fig. 9(c) and (d), are due to threshold 33 with granule size $2 \times 2$ and threshold 26 with granule size $6 \times 6$, respectively. Like the previous two images, the $T^{*}$ value increases/decreases with decrease/increase in granule size. Here we can see that while all the three output images are able to segment the water bodies (represented by the lower peak region in the histogram) from the rest of the objects, increase in $T^{*}$ value to 33 introduces more spurious (undesirable) regions (Fig. 9(d)), whereas decrease in $T^{*}$ value to 26 fails to detect some useful regions (e.g., airport runways, roads, canals) as object (Fig. 9(c)). This justifies the selection of 30 as the more appropriate threshold, and hence the choice of granule size $4 \times 4$.

\section{Conclusions}

Rough entropy of an image is defined using the concept of image granules. Based on this measure, 
a method of extracting object regions from an image is described by minimizing both object and background roughness. Here granules carry local information and reflect the inherent spatial relation of the image by treating pixels of a window as indiscernible or homogeneous. Maximization of homogeneity in both object and background regions during their partitioning is achieved through maximization of rough entropy; thereby providing optimum results for object-background classification. The guideline described for the choice of granule size from gray level distribution is seen to be appropriate. Extension of the algorithm to multi-class segmentation problem may constitute a part of future investigation.

It may be mentioned here that there exist some definitions of rough entropy useful for other applications. For example, the one defined by Beaubouef et al. (1999) is applicable to relational database and the one of Düntsch and Gediga (1998) for optimal granulation and feature selection. Reports are also available on image entropy measures and object extraction where image entropy is defined based on both histogram (Pun, 1981) and co-occurrence matrix (Pal and Pal, 1989) making use of the global and local information of image. Use of logarithmic and exponential gain functions in this regard is explained in (Pal and Pal, 1991).

\section{Acknowledgement}

A part of the work was completed while Prof. Pal held a Visiting Scientist position in the US Naval Research Lab, Washington, DC, USA under ONRIFO Grant No. N00014-04-1-4061. Mr. Uma Shankar acknowledges the project "Advanced techniques for remote sensing image processing" under the India-Trento Program for Advanced Research (ITPAR).

\section{References}

Beaubouef, T., Petry, F.E., Arora, G., 1999. Information measure for rough and fuzzy sets and application to uncertainty in relational database. In: Pal, S.K., Skowron, A. (Eds.), Rough Fuzzy Hybridization: A New Trend in Decision-making. Springer, Singapore, pp. 200-214.

Düntsch, I., Gediga, G., 1998. Uncertainty measures of rough set prediction. Artif. Intell. 106, 109-137.

Gonzalez, R.C., Woods, R.E., 2002. Digital Image Processing, second ed. Pearson Education Inc., Singapore.

Komorouski, J., Pawlak, Z., Polkowski, L., Skowron, A., 1999. Rough sets: A tutorial. In: Pal, S.K., Skowron, A. (Eds.), Rough Fuzzy Hybridization: A New Trend in DecisionMaking. Springer, Singapore, pp. 3-98.

Pal, S.K., 2001. Fuzzy image processing and recognition: Uncertainties handling and applications. Internat. J. Image Graphics 1 (2), 169-195.

Pal, N.R., Pal, S.K., 1989. Entropic thresholding. Signal Process. 16, 97-108.

Pal, N.R., Pal, S.K., 1991. Entropy: A new definition and its applications. IEEE Trans. Systems Man Cybernet. SMC-21, $1260-1270$

Pal, S.K., Mitra, P., 2002. Multispectral image segmentation using rough set initialized EM algorithm. IEEE Trans. Geosci. Remote Sensing 40, 2495-2501.

Pawlak, Z., 1991. Rough Sets, Theoretical Aspects of Reasoning about Data. Kluwer Academic, Dordrecht.

Pun, T., 1981. Entropic thresholding: A new approach. Comput. Graphics Image Process. 16, 210-239.

Skowron, A., Rauszer, C., 1992. The discernibility matrices and functions in information systems. In: Slowiński, R. (Ed.), Intelligent Decision Support, Handbook of Applications and Advances of the Rough Sets Theory. Kluwer Academic, Dordrecht, pp. 331-362.

Wojcik, Z., 1987. Rough approximation of shapes in pattern recognition. Comput. Vision Graphics Image Process. 40, 228-249.

Zadeh, L.A., 1997. Towards a theory of fuzzy information granulation and its centrality in human reasoning and fuzzy logic. Fuzzy Sets Systems 19, 111-127. 